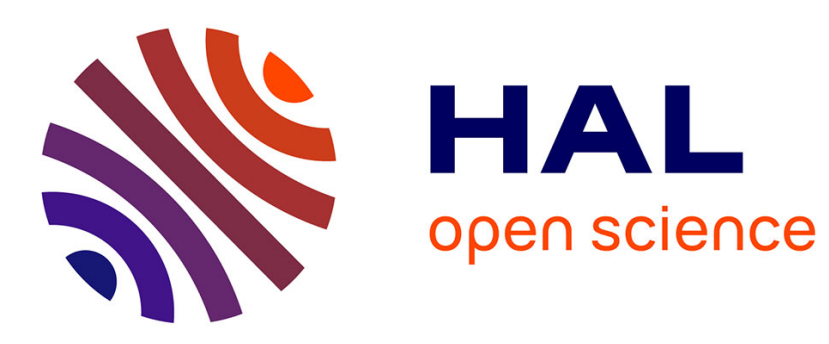

\title{
Acoustically Induced Flashback in a Staged Swirl-Stabilized Combustor
}

Corentin J Lapeyre, Marek Mazur, Philippe Scouflaire, Franck Richecoeur, Sébastien Ducruix, Thierry Poinsot

\section{- To cite this version:}

Corentin J Lapeyre, Marek Mazur, Philippe Scouflaire, Franck Richecoeur, Sébastien Ducruix, et al.. Acoustically Induced Flashback in a Staged Swirl-Stabilized Combustor. Flow, Turbulence and Combustion, 2017, 98 (1), pp.265 - 282. 10.1007/s10494-016-9745-2 . hal-01863807

\section{HAL Id: hal-01863807 https://hal.science/hal-01863807}

Submitted on 23 Jul 2020

HAL is a multi-disciplinary open access archive for the deposit and dissemination of scientific research documents, whether they are published or not. The documents may come from teaching and research institutions in France or abroad, or from public or private research centers.
L'archive ouverte pluridisciplinaire HAL, est destinée au dépôt et à la diffusion de documents scientifiques de niveau recherche, publiés ou non, émanant des établissements d'enseignement et de recherche français ou étrangers, des laboratoires publics ou privés. 


\title{
Acoustically Induced Flashback in a Staged Swirl-Stabilized Combustor
}

\author{
Corentin J. Lapeyre ${ }^{1,3} \cdot$ Marek Mazur² . \\ Philippe Scouflaire $^{2}$ - Franck Richecoeur ${ }^{2}$. \\ Sébastien Ducruix ${ }^{2}$ - Thierry Poinsot $^{3}$
}

\begin{abstract}
This paper describes a joint experimental and numerical investigation of the interaction between thermoacoustics and flashback mechanisms in a swirled turbulent burner. An academic air/propane combustor terminated by a choked nozzle is operated up to 2.5 bars. Experiments show that the flame can stabilize either within the combustion chamber or flashback inside the injection duct, intermittently or permanently. The present study focuses on the mechanisms leading to flashback: this phenomenon can occur naturally, depending on the swirl level which can be adjusted in the experiment by introducing axial flow through the upstream inlet. It can also be triggered by acoustic waves, either through acoustic forcing or self-excited thermoacoustic instability. Flashback is difficult to study experimentally, but it can be investigated numerically using LES: in a first configuration, the outlet of the chamber is treated as a non-reflecting surface through which harmonic waves can be introduced. In this case, a $20 \mathrm{kPa}$ acoustic forcing is sufficient to trigger permanent flashback after a few cycles. When the LES computational domain includes the choked nozzle used experimentally, no forcing is needed for flashback to occur. Self-excited oscillations reach high levels rapidly, leading to flame flashback, as observed experimentally. These results also suggest a simple method to avoid flashback by using fuel staging, which is then tested successfully in both LES and experiments.
\end{abstract}

Keywords Flashback · Flame stabilization · Thermoacoustic instabilities · Experimental combustion · LES

Corentin J. Lapeyre

corentin.lapeyre@imft.fr

1 CERFACS, 42 Av. G. Coriolis, 31057, Toulouse Cedex, France

2 Laboratoire EM2C, CNRS, Centrale-Supélec, Université Paris-Saclay, Grande Voie des Vignes, 92295, Châtenay-Malabry Cedex, France

3 IMF Toulouse, UMR CNRS/INP-UPS 5502, Allée du Pr. C. Soula, 31400, Toulouse Cedex, France 


\section{Introduction}

The development of modern aeronautical combustion processes presents several challenges. Combustion instabilities are one of them [1-4]. Another one is the control of flashback, where the flame stabilizes upstream of the chamber, in an undesired position [5-8]. These two intrinsically unsteady mechanisms are not independent $[9,10]$. The present study describes a joint experimental and numerical investigation of the links between flashback and instabilities in a laboratory scaled swirled combustor.

Flashback occurs when the flame front can propagate upstream of the chamber. This can happen in any system if the laminar or turbulent flame speed exceeds the local flow speed. Other less trivial mechanisms can also cause the flame to flash back, even if this condition is not met [11]. Two issues are commonly distinguished in this field: (1) the reason for flashback to start, i.e. the mechanism through which the flame propagates upstream from its design position; and (2) the propagation and/or continous presence of the flame in the injection duct. Both aspects are critical for burner robustness: (1) the flame should remain in the combustion chamber during operation, and (2) if the flame momentarily travels upstream into the injection duct, it should rapidly recede back to its design position.

Three main mechanisms can trigger flame flashback [11-13]: ${ }^{1}$

1. Wall boundary layer flashback (WBLF) Since flow velocities go to zero at the walls, flames can flashback in near-wall zones. This type of flashback is controlled by the velocity gradient at the wall $[5,14,15]$. WBLF is unlikely in swirled systems, as the swirled flow induces maximum velocity and large velocity gradients close to the walls. In practice, it has not been observed in the context of this study, and will not be further discussed.

2. Thermoacoustic instabilities Early experiments [9] described a backward facing step in which the flame stabilization position was strongly influenced by self-excited acoustic waves: for a given set of boundary acoustic impedances, a self-sustained thermoacoustic instability could trigger intermittent flame flashback. This behavior was also observed with active acoustic excitation [16]. The flashback mechanism in this case is simple: the acoustic oscillation leads to velocity perturbations larger than the mean flow, thus inducing flow reversal at the flame location. This flashback may oscillate at the thermoacoustic frequency (intermittent flashback) or lead the flame to stabilize permanently at another location (permanent flashback).

3. Combustion induced vortex breakdown (CIVB) Swirl is the most common stabilization method in combustion chambers [17]. The recirculation zone induced by swirl [18] contains burnt gases which stabilize the flame but also open the path for flame flashback in this region of reverse velocities [8, 19, 20].

Mechanisms 1, 2 and 3 can all force the flame to flashback and enter the duct upstream of the chamber. Once the flame has flashed back, it can stabilize upstream permanently. This can happen $e . g$ if a component has a wake that is weak at the dump plane but strong upstream [11]. If the flow is swirled, flashback can also take place along a high velocity vortex core axis [13]. Strong swirl decreases the pressure upstream on the axis and the local

\footnotetext{
${ }^{1}$ In certain combustors, where the fresh gases are compressed up to high pressures and temperatures, flashback can also occur because of autoignition in the injection system. This case is not considered here, because the low temperature of the fresh charge prevents autoignition in all cases.
} 
flow velocity, offering a route for the flame to continue to propagate upstream. The propagation velocities observed in this case often exceed typical flame displacement speeds, and the mechanism controlling this type of flashback is not well understood [21-24]. It involves a radial pressure gradient and an axial density gradient which induce a negative velocity leading to upstream propagation. The dependency on density gradient has been investigated using experiments $[25,26]$ and DNS $[27,28]$. In a real gas turbine chamber, several flashback mechanisms may appear, combine and lead to unexpected flashback. In the present study, a pressurized swirled chamber is analyzed using experiments and LES. This system exhibits violent and intermittent as well as permanent flashback. LES suggests that the onset of these flashbacks is due to a combination of mechanisms 2 and 3, namely acoustic pulsations and CIVB. LES shows that the combustor is robust to some extent to intermittent flashback created by acoustic fluctuations, but that, under sufficient acoustic forcing, it can exhibit permanent flashback through the high-velocity vortex core. The resulting phenomenon is called Acoustically Induced CIVB, or AI-CIVB hereafter. AI-CIVB may occur even though the setup is robust to CIVB.

Experimental results are discussed in Section 2, showing that premixed cases systematically lead to flashback for the regime studied here while fuel staging in the swirler passages allows to suppress flashback. To explain this behavior, a numerical approach using LES is chosen (Section 3). Section 4 describes LES where the outlet pressure is pulsated to assess flashback robustness to acoustic forcing. Once this behavior under external forcing is determined, a simulation with a choked nozzle outlet (Section 5) shows that self-excited oscillations arise and lead to flashback, as predicted by the experiments. In Section 6, LES confirms that fuel staging inhibits flashback, and yields insight into the underlying mechansim.

\section{Experimental Study of the Swirl-Stabilized Model Combustor}

The CESAM-HP test bench located in EM2C Laboratory is a pressurized combustor designed for lean premixed swirled combustion of gaseous propane (Fig. 1). The injection system contains two stages with tangential injection stages where premixed air and propane are fed tangentially. Air staging between the two injection has a strong influence on flashback. Fuel staging controls the equivalence ratio pattern reaching the flame, hence the flame stabilization. The left inlet of the duct is terminated by a system called the Impedance Control System (ICS). This device is a perforated plate with optimal porosity and bias flow,

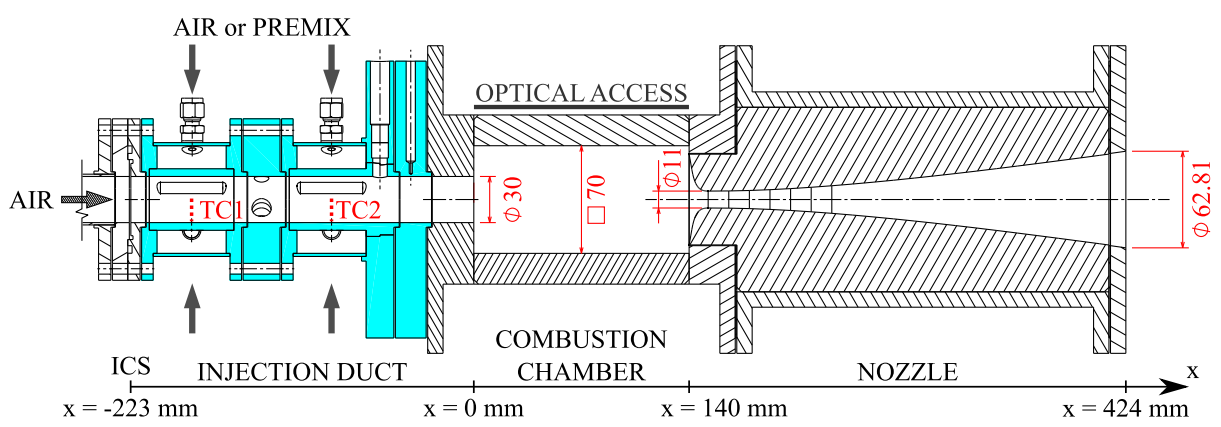

Fig. 1 Schematic of the CESAM-HP experimental setup 
backed by a cavity of controllable length, offering control over the acoustic impedance of the upstream end [29-31]. While constituting only a small part of the total flow, the axial bias flow affects swirl and therefore flashback [32, 33].

The combustion chamber has a $70 \times 70 \mathrm{~mm}$ square section large enough to avoid excessive flame-wall interaction. The chamber is long enough to avoid contact between the flame and the nozzle. Quartz windows allow optical diagnostics in the combustion chamber. The chamber walls are cooled so that piezo-resistive sensors (Kistler 4045A) can be used to measure static and dynamic pressures [34-36].

The exhaust nozzle is a classical Laval nozzle accelerating the burnt gases and increasing the mean pressure in the chamber. For flow rates higher than $14.5 \mathrm{~g} / \mathrm{s}$, the nozzle is choked and the chamber pressure reaches values up to 2.5 bar.

The design of this chamber has a specificity: it is prone to flame flashback. The tangential premixed injection leads to a strong rotational motion, characterized by a high swirl value (Section 3.2). Previous studies have shown that the recirculation zone associated with this kind of flame shape is exposed to vortex breakdown, leading to upstream flame propagation [37-39]. Many practical chambers use swirling flows for flame stabilization and a central plug, which prevents flashback. Here the absence of a plug in the injector allows the flame to propagate upstream more easily [13].

\subsection{Operating conditions}

The combustor is fed with cold pressurized air at $300 \mathrm{~K}$ and 6 bar. Mass flow rates are fixed via 3 choked mass flow controllers and can range from 3 to $20 \mathrm{~g} \mathrm{~s}^{-1}$ (Table 1). The pressure in the chamber is controlled by the total flow rate and the outlet nozzle diameter. The latter is choked for flow rates higher than $14.5 \mathrm{~g} \mathrm{~s}^{-1}$. The bench is designed to operate up to 2.5 bar, under lean combustion with equivalence ratios $\phi$ ranging from 0.5 to 1 .

Two operating points have been chosen for this study: a PREMIXED one where air and fuel are equally distributed between both tangential injection stages; and a STAGED point where all the fuel is fed through the downstream injector. The axial airflow has the

Table 1 Chosen operating conditions for experimental study

\begin{tabular}{lll}
\hline & PREMIXED & STAGED \\
\hline$p_{u}^{a}$ & $6 \mathrm{bar}$ & $6 \mathrm{bar}$ \\
$p_{u}^{f}$ & $6 \mathrm{bar}$ & $6 \mathrm{bar}$ \\
$T_{u}^{a}$ & $300 \mathrm{~K}$ & $300 \mathrm{~K}$ \\
$T_{u}^{f}$ & $300 \mathrm{~K}$ & $300 \mathrm{~K}$ \\
$\dot{m}_{1}^{a}$ & $8.5 \mathrm{~g} \mathrm{~s}^{-1}$ & $7 \mathrm{~g} \mathrm{~s}^{-1}$ \\
$\dot{m}_{2}^{a}$ & $8.5 \mathrm{~g} \mathrm{~s}^{-1}$ & $10 \mathrm{~g} \mathrm{~s}^{-1}$ \\
$\dot{m}_{a x}^{a}$ & $1 \mathrm{~g} \mathrm{~s}^{-1}$ & $1 \mathrm{~g} \mathrm{~s}^{-1}$ \\
$\dot{m}_{1}^{f}$ & $0.51 \mathrm{~g} \mathrm{~s}^{-1}$ & $0 \mathrm{~g} \mathrm{~s}^{-1}$ \\
$\dot{m}_{2}^{f}$ & $0.51 \mathrm{~g} \mathrm{~s}^{-1}$ & $0.97 \mathrm{~g} \mathrm{~s}^{-1}$ \\
$\Phi^{\text {global }}$ & 0.9 & 0.85 \\
$p^{\text {chamber }}$ & $2.27 \mathrm{bar}^{*}$ & $2.27 \mathrm{bar}^{a}$ \\
Power & $46.8 \mathrm{~kW}$ & $45.0 \mathrm{~kW}$ \\
\hline
\end{tabular}

a: air, f: fuel, u: unburnt, b: burnt * estimated value 
same value for the STAGED and the PREMIXED point. The two operating points are summarized in Table 1.

\subsection{Experimental procedure}

First experimental tests showed that the PREMIXED case leads to permanent flashback, while the STAGED case allows to avoid flashback. Because this bench was not designed for flashback studies initially, the PREMIXED regime could not be sustained for long periods.

Normalized mean $\mathrm{OH}^{*}$ emission images are presented in Fig. 2 for the two operating points. $\mathrm{OH}^{*}$ levels are normalized by the highest local intensity of both cases. The images were not Abel-transformed due to the significant asymmetry of the mean flames.

The PREMIXED case (Fig. 2(a)) shows low level OH* emissions coming mainly from the plume of the flame. No clear flame front can be identified. The mean $\mathrm{OH}^{*}$ images show either burnt gases or the intermittent presence of the flame in the chamber, suggesing that the PREMIXED flame has flashed back and is stabilized in the upstream duct $(x<0)$.

In the STAGED case (Fig. 2(b)) a sharp flame front can be observed starting at the lips of the injection system. The $\mathrm{OH}^{*}$ emission levels are much stronger than those observed in the PREMIXED case, showing that the flame is located permanently in the combustion chamber.

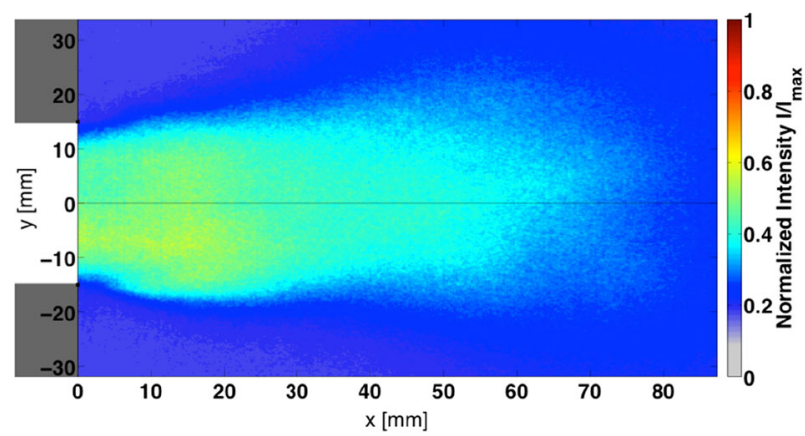

(a) PREMIXED

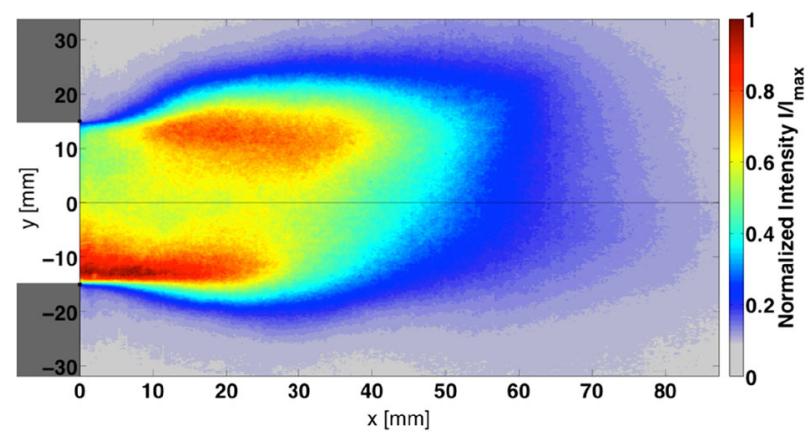

(b) STAGED

Fig. 2 The mean $\mathrm{OH}^{*}$ emission images show the different flame shapes of the two operating points. The intensity values are absolute direct line-of-sight measurements by the ICCD camera, normalized by the maximum of both operating points 
Table 2 The temperature profiles (in ${ }^{\circ} \mathrm{C}$ ) in the admission stage indicate the flashback depth

\begin{tabular}{llllll}
\hline & \multicolumn{2}{l}{ PREMIXED } & & \multicolumn{2}{l}{ STAGED } \\
\cline { 2 - 3 } $\mathrm{r}[\mathrm{mm}]$ & $\mathrm{TC} 1$ & $\mathrm{TC} 2$ & & $\mathrm{TC} 1$ & TC2 \\
\hline 0 & 1021 & 1203 & 28 & 621 \\
5 & 1171 & 1362 & 29 & 607 \\
10 & 1012 & 1152 & 28 & 598 \\
15 & 852 & 841 & 24 & 575 \\
\hline
\end{tabular}

Temperature measurements in the injection duct (TC1 and TC2 Fig. 1) confirm these observations (Table 2). In the PREMIXED case, the temperature in both injection ducts exceeds $1000^{\circ} \mathrm{C}$, indicating that the flame has flashed back permanently into the injection tubes. In the STAGED setup, on the other hand, the first injection stage remains cold. The second one, closer to the chamber, reaches $600^{\circ} \mathrm{C}$, indicating either intermittent flame presence or lower mean gas temperature. For the PREMIXED point, flashback is so intense that the chamber cannot be operated for more than a few minutes because the injector walls are not water-cooled. Thermocouple measurements are a crude method of determining flame position, but the combination of multiple thermocouples has been used successfully in previous work for a rough localization of the flashback depth [40, 41]. Flashback through the entire injection duct is also confirmed a posteriori by inspecting the perforated plate of the ICS on the upstream part of the device. This plate is covered with soot, indicating that it has been in contact with the flame.

To elucidate the mechanism leading to flashback, experiments in the EM2C combustor were found to be difficult and sometimes dangerous. A fully numerical approach was therefore preferred, as described in the next sections.

\section{LES Setup for the CESAM-HP Combustor}

The fully compressible explicit code AVBP is used to solve the filtered multi-species 3D Navier-Stokes equations with simplified thermochemistry on unstructured meshes [42, 43]. A third-order in space and time Taylor-Galerkin finite element scheme called TTGC [44] is used to accurately propagate vortices and acoustic waves. The NSCBC approach [45] with transverse terms corrections [46] is used for boundary conditions. The subgrid-scale stress model is the Sigma model [47] and walls are treated as no-slip adiabatic interfaces. A Thickened Flame strategy with the Charlette-Meneveau efficiency function [48] is used to resolve the flame on the grid and model turbulence-flame interactions. Chemical kinetics are modeled using a global 1-step scheme representing the global reaction between propane and air. One-dimensional flame computations were used to verify that this global scheme predicted flame speeds and adiabatic flame temperatures correctly for the range of conditions encountered in this study [49].

\subsection{Numerical test cases}

Two configurations were retained for numerical simulations (Table 3) : 
Table 3 Numerical setups for the CESAM-HP test bench

\begin{tabular}{llllll}
\hline & \multicolumn{2}{l}{ OPEN Setup (Fig. 3(b)) } & & \multicolumn{2}{l}{ CHOKED Setup (Fig. 3(a)) } \\
\cline { 2 - 3 } Case name & OPEN-NR & OPEN-FO & & CHOKED-PR & CHOKED-ST \\
\hline Domain & OPEN & OPEN & & CHOKED & CHOKED \\
Outlet & Non-reflecting & Acoustic forcing & Choked nozzle & Choked nozzle \\
Operating Point & PREMIXED & PREMIXED & PREMIXED & STAGED \\
Exp. data & NO & NO & & YES & YES \\
ICS Flow & Premixed & Premixed & & Premixed & Air \\
\hline
\end{tabular}

- the CHOKED (Fig. 3(a)) case, where the domain includes the nozzle and is cut after the geometric throat in the nozzle. This nozzle termination behaves almost like an acoustic wall $\left(u^{\prime}=0\right)$ [50] and is expected to generate strong reflections and possible unstable modes. This LES domain corresponds to the geometry used experimentally;

- the OPEN (Fig. 3(b)) case, where the domain is cut upstream from the nozzle to control the acoustic behavior of the outlet as well as to introduce acoustic forcing through the outlet section. To do this, a characteristic treatment of the boundary conditions is used to provide either a non-reflecting outlet condition, or a forced one with an upstream acoustic wave. This configuration was not reproduced experimentally.

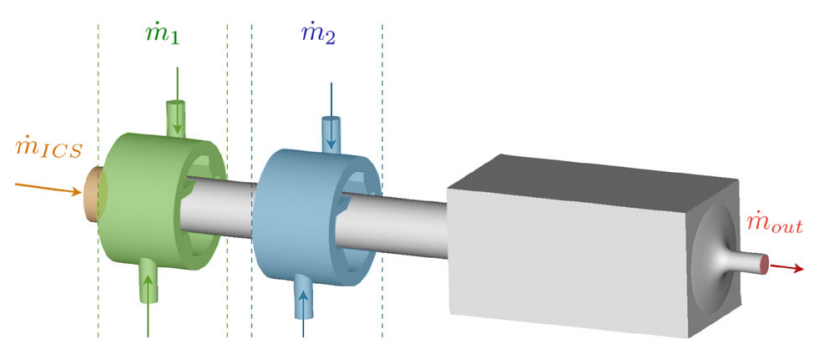

(a) CHOKED setup

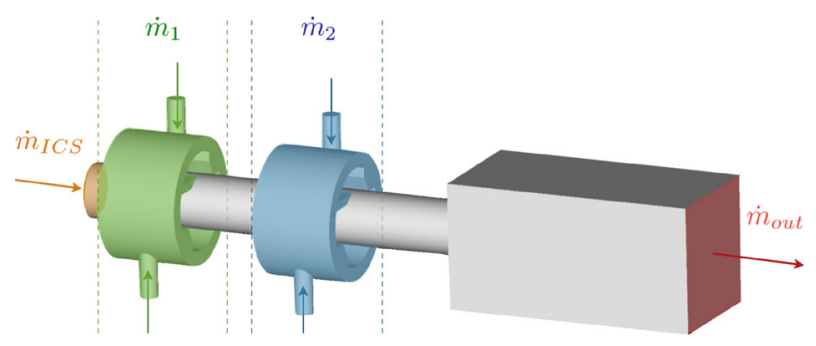

(b) OPEN setup

Fig. 3 LES configurations 


\subsection{Swirl control}

The parameter controlling the overall swirl is the flow split between ICS ( $\dot{m}_{I C S}$ ) and the two swirled injectors $\left(\dot{m}_{1}, \dot{m}_{2}\right)$. The flow through the ICS is not swirled, whereas the flow through the two plenums is strongly swirled. As a result, the staging balance between the ICS and the plenums controls the flow swirl at the dump plane. Swirl is measured in the simulation according to:

$$
S w=\frac{\iint_{(S)} \rho U(r W) d S}{R \iint_{(S)} \rho U^{2} d S}
$$

where $S$ is a plane normal to the flow, $R$ is the duct radius, $U$ and $W$ are the axial and azimuthal velocities, respectively, and $\rho$ is the fluid density.

Figure 4 shows the swirl number as a function of axial position for a case with no flow through the ICS in non-reacting conditions.

Three different regions can be identified between the last injector and the dump plane:

- $\quad 70 \mathrm{~mm}$ to $-45 \mathrm{~mm}$ : the flow swirl is not yet established after the second swirl plenum:

- $45 \mathrm{~mm}$ to $-15 \mathrm{~mm}$ : swirl decreases approximately linearly with axial position, as expected from previous studies [51];

- $\quad 15 \mathrm{~mm}$ to $-0 \mathrm{~mm}$ : vortex breakdown in the chamber starts modifying the swirl.

As a result, the swirl that would be achieved at the dump plane if no vortex breakdown occurred in the chamber is higher than the one measured directly at that position. The swirl measured $10 \mathrm{~mm}$ before the dump plane is a good measure of this theoretical value. For this reason, the reference position for all swirl estimations hereafter is $x=-10 \mathrm{~mm}$.

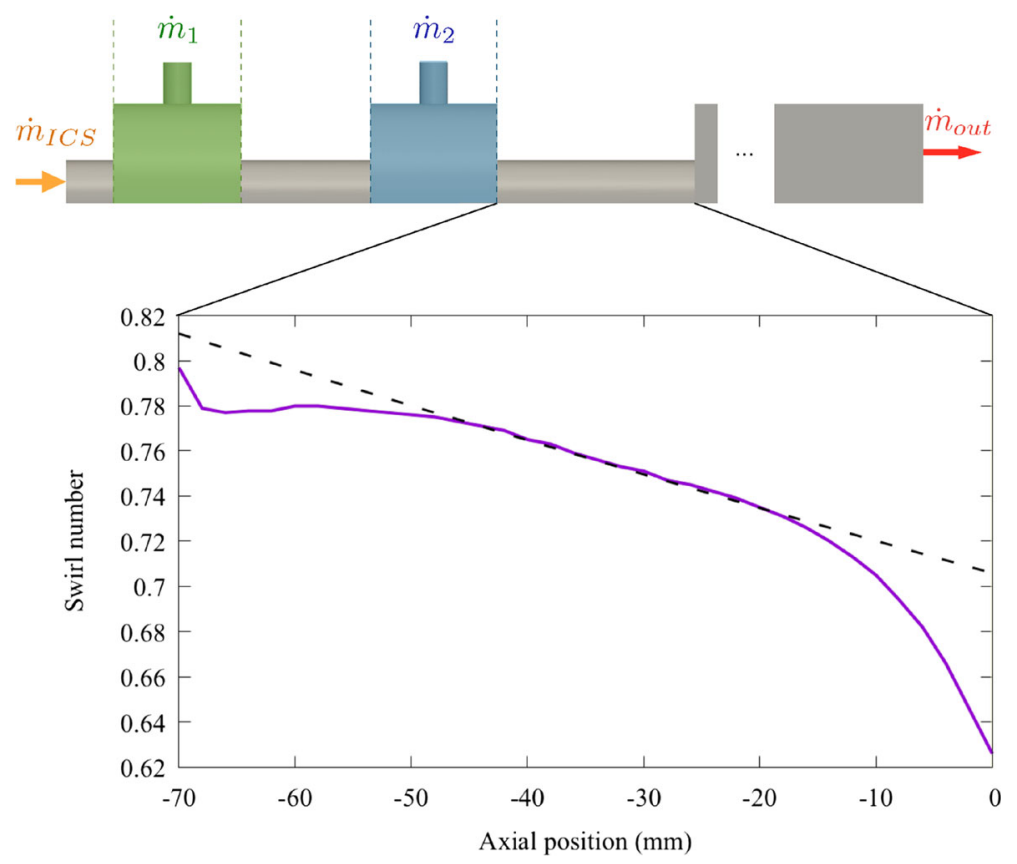

Fig. 4 Solid line: time-averaged swirl number along the injection tube taken from LES. Dotted line: linear fit during established decreasing swirl. Dump plane is at $x=0$ 


\section{OPEN Outlet Numerical Investigation}

While the experiment indicates that flashback occurs for the PREMIXED case and not for the STAGED case, it does not give any information on the mechanisms controlling flashback. In this section, LES was used to tackle this question. First, the configuration without nozzle (OPEN-NR, Section 4.1) was studied. Then, this open setup was forced acoustically (OPEN-FO, Section 4.2) to check whether flashback can be caused by acoustic oscillations and which forcing level is required to trigger it. These configurations cannot be operated experimentally.

\subsection{OPEN-NR test case: unforced flashback}

LES was used first in the OPEN-NR case where the outlet is non-reflecting and no acoustic instability can grow. The ICS mass flow rate is varied between 0 and $20 \%$. The following swirl-control strategy is used: the total mass flow rate is kept constant while the value of mass flow rate through the ICS is varied between 0 and $20 \%$ of the total mass flow rate. Each case is labelled as ICSXX where XX is the percentage of mass flow rate through the ICS. The two experimental configurations have a 5.6\% ICS mass flow rate.

Table 4 lists the swirl numbers measured for each flame and the overall stabilization behavior.

Two flame stabilization positions are observed:

- for ICS10 and ICS20, a typical 'M' shaped flame is stabilized inside the combustion chamber, referred to as a Chamber Stabilized (CS) flame (Fig. 5(a));

- for ICS00 and ICS05, the flame eventually propagates upstream all the way to the ICS. It then stabilizes in this position, referred to as the Fully Flashed Back (FFB) position (Fig. 5(b)).

As expected, swirl directly impacts flame stabilization. Results reveal that the critical swirl number leading to flashback with a non-reflecting outlet is between 0.64 and 0.7 .

A first conclusion is that for the OPEN-NR case, $5 \%$ mass flow rate through the ICS is not enough to prevent flashback. $10 \%$ however is sufficient. This conclusion is valid in the absence of acoustic activity because LES allows to create a quiet case by using a nonreflecting outlet. The next question is whether acoustic oscillations can affect flashback Section 4.2.

\subsection{OPEN-FO: stabilization robustness}

In order to evaluate the robustness of the flame stabilization to acoustic forcing, the OPEN setup in the ICS10 case $10 \%$ of the flow is fed through the ICS) is perturbed at the exit

Table 4 Flame stabilizing position as a function of the flow rate through the ICS $\dot{m}_{I C S}$, and the swirl number Sw

\begin{tabular}{lllll}
\hline Case name & ICS00 & ICS05 & ICS10 & ICS20 \\
\hline$\dot{m}_{\text {ICS }}$ & 0 & 0.05 & 0.1 & 0.2 \\
Swirl number & 0.71 & 0.70 & 0.64 & 0.62 \\
Flashback $?$ & Yes & Yes & No & No \\
\hline
\end{tabular}




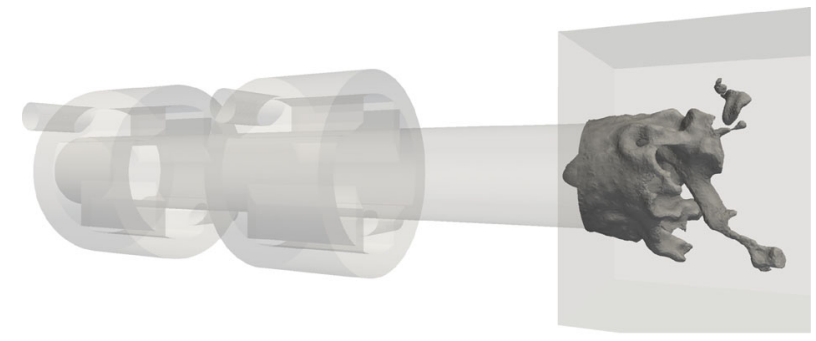

(a) Chamber stabilized (CS) flame

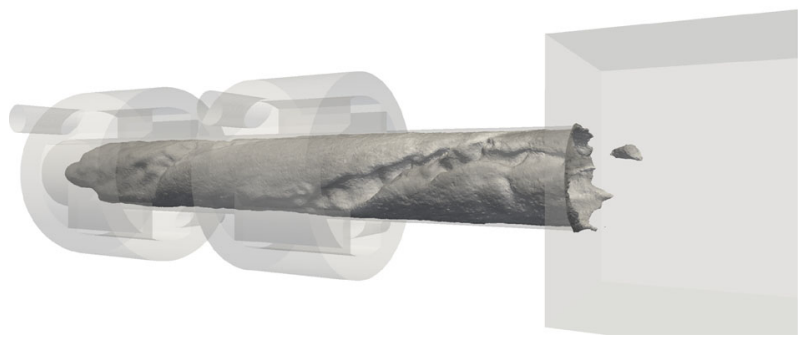

(b) Fully flashed back (FFB) flame

Fig. 5 Views of the flame ( $T=1000 \mathrm{~K}$ isosurface) in the two stabilization positions observed in the LES: (a) Chamber stabilized (CS), and (b) Fully flashed back (FFB)

plane with a harmonic acoustic perturbation. This wave travels upstream, changes the local velocity and excites the flame.

Starting from the naturally robust ICS10 case, two excitation strengths are imposed: 10 $\mathrm{kPa}$ (ICS10-10k) and $20 \mathrm{kPa}$ (ICS10-20k). The forcing frequency $(200 \mathrm{~Hz}$ ) corresponds roughly to the first acoustic mode of the CHOKED configuration because this is the mode expected to be active in the experiment. The flame is fully flashed back (FFB) for ICS1020k. For ICS10-10k, a third state appears where the flame oscillates between chamber

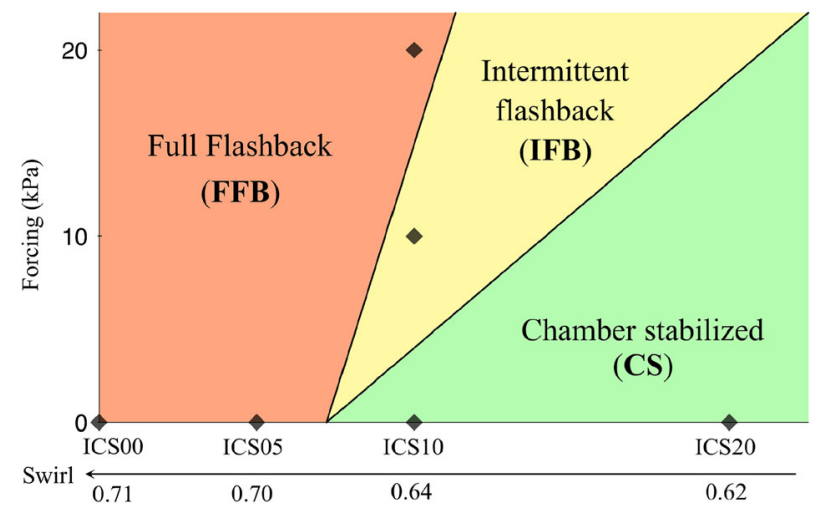

Fig. 6 Flame states for the OPEN setup versus swirl $S w$ and forcing amplitude (in $\mathrm{kPa}$ ). Symbols indicate LES data 


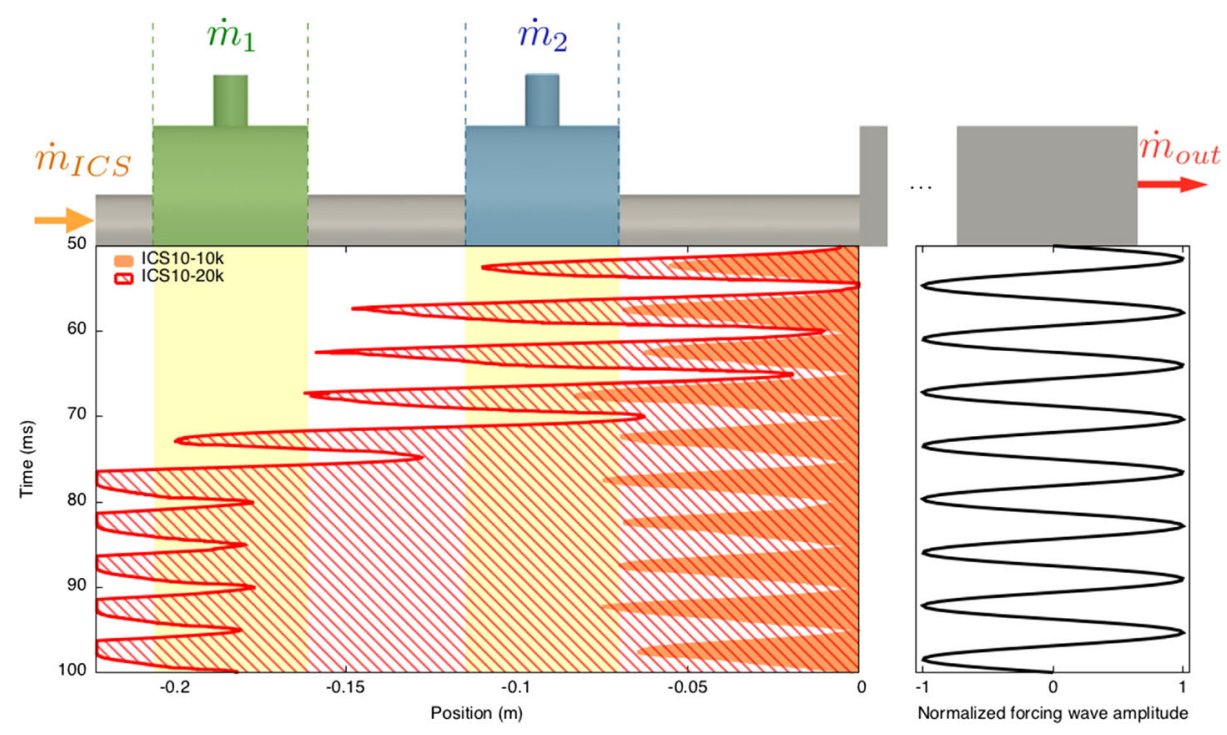

Fig. 7 Flame leading point position versus time for two acoustic forcing strengths: ICS10-10k and ICS10$20 \mathrm{k}$. Time as ordinate, going down

stabilized (CS) and partially flashed back. This is called Intermittent FlashBack (IFB) in this paper. Fig. 6 gives a schematic map of flame stabilization versus swirl and forcing.

\subsection{Flame leading point position and speed}

During simulations, the most upstream flame tip position (i.e. the $1000 \mathrm{~K}$ isosurface, called here the leading point) can be recorded versus time. In all CS cases, the flame front remains in the chamber, and the leading point remains close to the dump plane position $x_{d u m p}=0$. With acoustic forcing, the flame recedes in the injection duct. Leading point positions (abscissa) versus time (ordinate, going down) are shown for each forcing level in Fig. 7. ICS10-10k shows that a strong acoustic wave is able to affect the flame, resulting in intermittent flashback (IFB), but unable to force a permanent full flashback (FFB): the leading point enters the swirl tube in phase with the negative velocity perturbations resulting from the acoustic wave which carry the flame upstream (Fig. 7). However, it exits the tube when

Fig. 8 Leading point position versus time for ICS10-20k. Two separate flame speeds are observed: a very fast intermittent speed $v_{\text {IFB }}$ and a slower drift to full flashback $v_{\text {FFB }}$

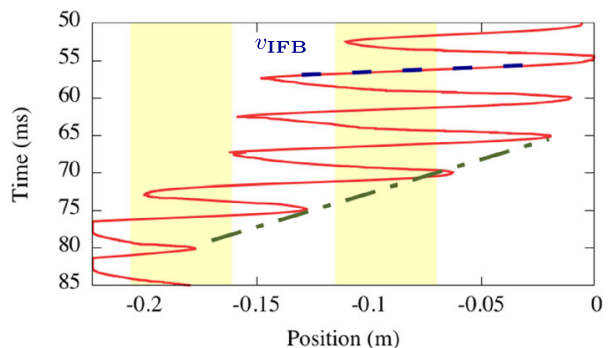


the acoustic velocity changes sign, and its upstream position (from the dump plane) never exceeds $7 \mathrm{~cm}$ in the tube.

A different behavior is observed for ICS10-20k (Fig. 8). Two different flame propagation speeds are involved:

- during the first instants, IFB is observed, as the flame is intermittently convected in and out of the injection duct, in phase with the pressure forcing, at a maximum velocity $v_{\text {IFB }} \approx 60$ to $70 \mathrm{~m} \mathrm{~s}^{-1}$ which is of the order of the acoustic velocity perturbation;

- then, the flame progressively recedes in the swirl tube until it reaches the ICS and stabiles there $(\mathbf{F F B})$. This drift occurs at a lower velocity $v_{\mathbf{F F B}} \approx 10$ to $15 . \mathrm{m} \mathrm{s}^{-1}$

The $v_{\text {IFB }}$ velocities observed in both ICS10-10k and ICS10-20k are coherent with acoustic displacement speeds, and scale with the forcing amplitude. The drift velocity $v_{\mathbf{F F B}}$, however, is lower, of the same order as the maximum azimuthal velocity of the cold flow in the injection duct. This is coherent with experimental measurements of flame propagation speeds along a vortex axis in swirled ducts $[25,52]$. Observations of the flame shape during IFB and FFB (Fig. 9) also show differences:

- during IFB, the flame fills the entire injection duct up to the walls;

- during FFB the leading point is trapped in the vortex core, and propagates along its axis.

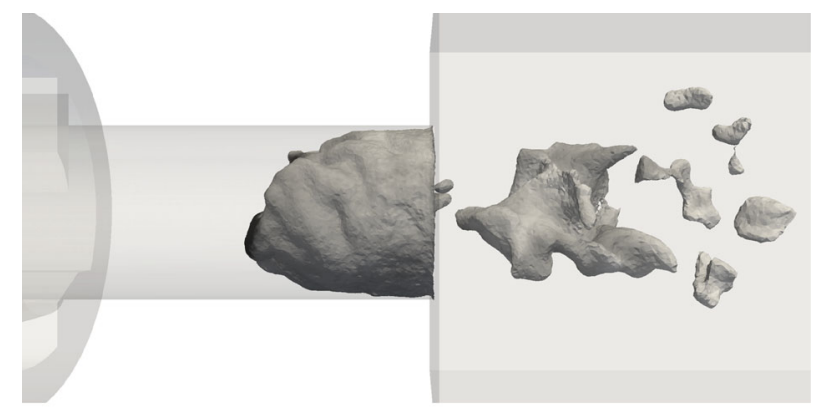

(a) IFB during recession in the injection.

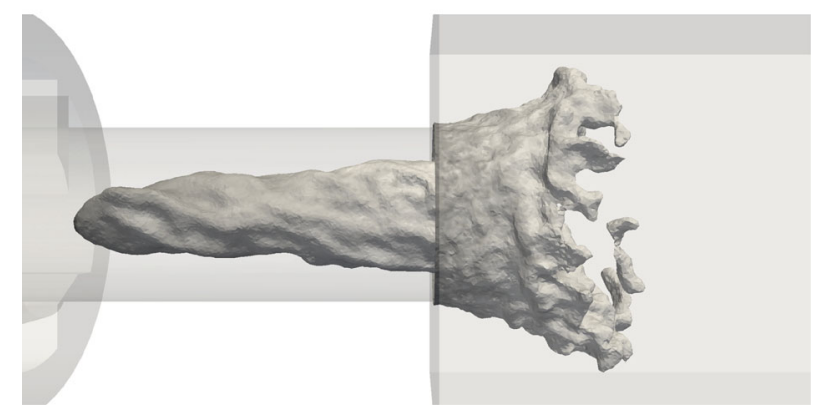

(b) FFB in the early stages of drift.

Fig. 9 Views of the flame ( $T=1000 \mathrm{~K}$ isosurface) during (a) the IFB phase, and (b) in the early drift stage of FFB 
Since, for ICS10, vortex core propagation only appears when combined with acoustic perturbation, this suggests that a mechanism called here Acoustically Induced CIVB (AICIVB) is responsible for flashback of this setup. AI-CIVB is observed here with a controlled acoustic forcing introduced at the outlet. However it also occurs in a self-excited mode as shown in Section 5.

\section{Flashback Induced by Self-Excited Instabilities in the Choked Case (CHOKED-PR)}

The previous section has shown that the flame can flashback when acoustic oscillations are imposed at a sufficiently high level. In the CHOKED LES setup as in the experiment, no forcing is applied but self-excited oscillations can occur because of acoustic reflections on the nozzle and self-excited instabilities. The LES of the CHOKED-PR case includes the nozzle. Since it is choked, no acoustic information can travel back through this supersonic section and the acoustic behavior of the experimental chamber is entirely reproduced as soon as the sonic line is included in the domain.

A 90 ms LES run of the CHOKED-PR test case with ICS10 flow split (Fig. 10) shows that the flame oscillates and then flashes back. The leading point position and mean chamber pressure versus time are correlated: the flame is initially stabilized at the dump plane; pressure oscillations then grow, leading to intermittent flashback. When pressure oscillations reach a critical level, full flashback is triggered. The mechanism is similar to the OPENFO case forced at $20 \mathrm{kPa}$ with an important difference: no forcing is applied here and the acoustic waves leading to CIVB are self-excited by the thermoacoustic instability. The flame evolution during this self-excited flashback can be described in three phases: initial growth, thermoacoustic instability and flashback.
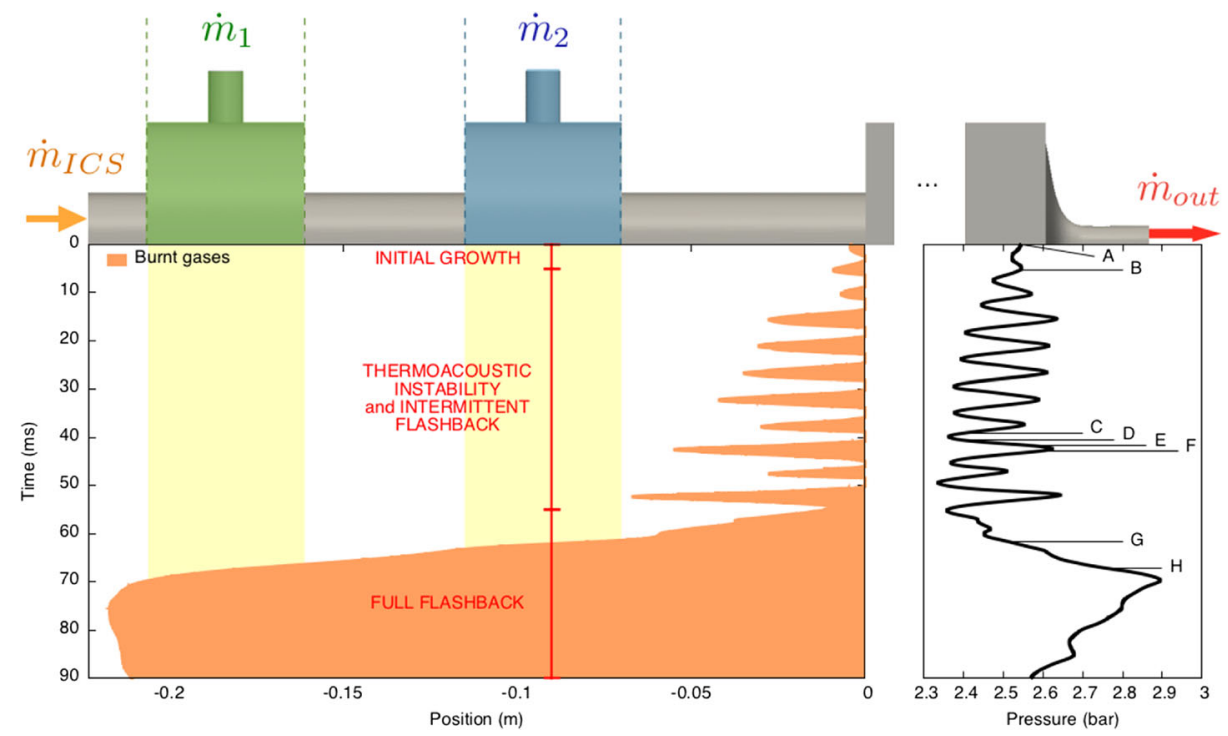

Fig. 10 Flame position versus time for ICS10 CHOKED-PR configuration. Chamber pressure is displayed on right side. Snapshot positions of Fig. 11 are marked by letters A to $\mathrm{H}$ 

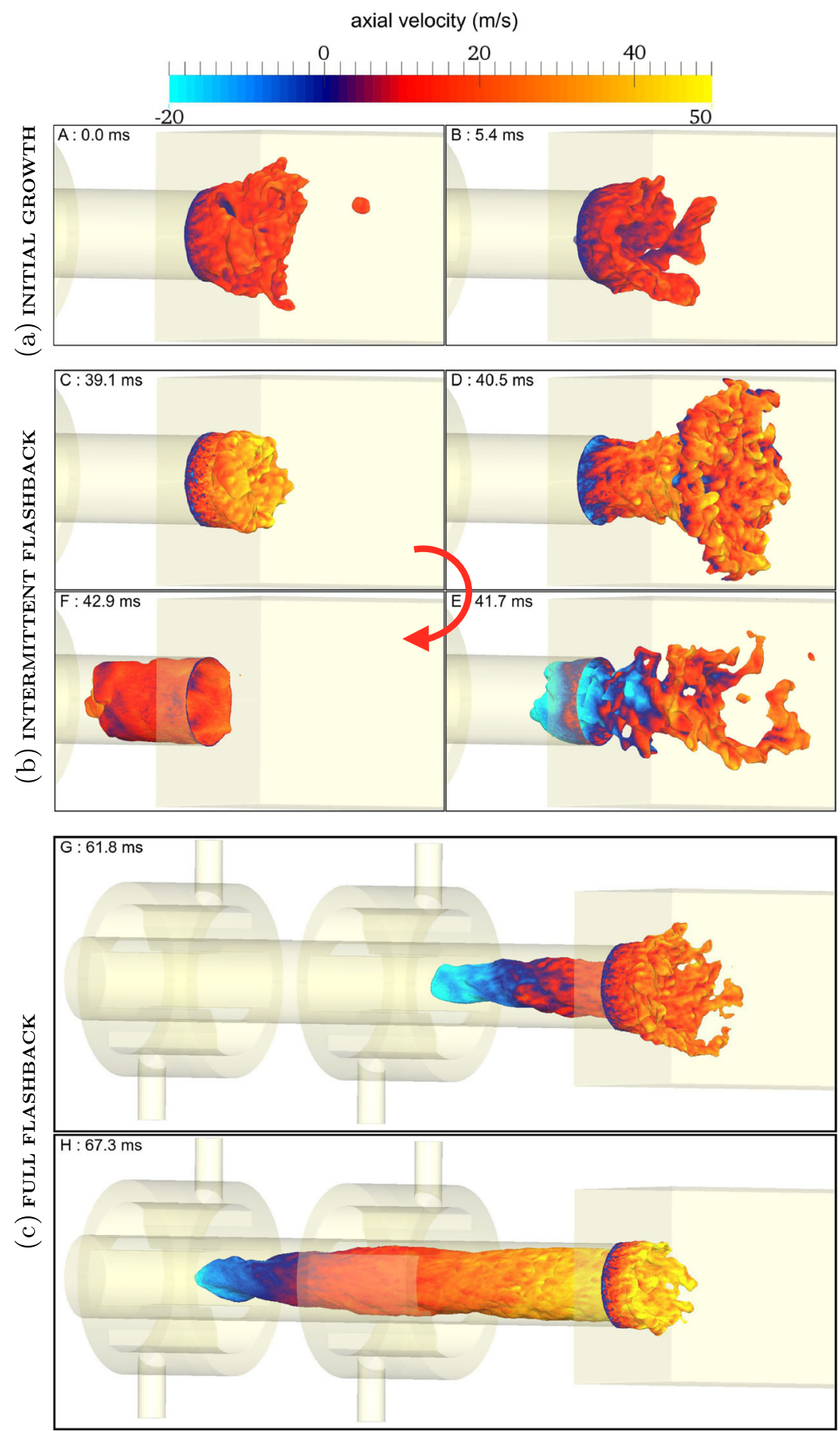

Fig. $111000 \mathrm{~K}$ isosurface colored by axial velocity, during the initial growth (top), intermittent flashback (center) and full flashback (bottom) phases 
Initial growth The solution of the OPEN-NR case is used to initialize a flame without thermoacoustic activity. During the first $5 \mathrm{~ms}$, the pressure oscillations are very weak, and the flame is stabilized in the chamber (Fig. 11.A). Pressure oscillations grow slowly at a frequency of $188 \mathrm{~Hz}$.

Thermoacoustic instability After $5 \mathrm{~ms}$, pressure oscillations increase more rapidly, and the flame motion amplifies (Fig. 10). After $40 \mathrm{~ms}$, the flame undergoes high amplitude variations in position and surface (Fig. 11.B). This self-excited mode still oscillates at 188 $\mathrm{Hz}$, as determined using the autocorrelation of pressure fluctuations at a reference point in the swirl tube. This behavior is sustained for approximately $50 \mathrm{~ms}$ (10 cycles). Since the flame recedes far into the swirl tube but complete flashback is not triggered yet, it is called intermittent flashback (IFB).

Flashback When pressure oscillations reach a critical level ( $t=60 \mathrm{~ms}$ in Fig. 10), the flame enters the swirl tube but does not exit it anymore. The leading point recedes gradually towards the ICS in $15 \mathrm{~ms}$. Fig. 11.C displays two views of the flame during the flashback phase. The leading point velocity during this phase is $13 \mathrm{~ms}^{-1}$ on average. This result agrees with VCF theories [52]: flame propagation along a vortex axis is a peculiar phenomenon in which the flame velocity scales with the azimuthal velocity and can be much higher than a simple laminar or even turbulent flame speed. It also scales well with the drift velocity $v_{\mathbf{F F B}}$ found in the OPEN-FO case.

Figure 10 confirms the link between flame recession in the swirl tube and acoustic activity: flashback is triggered at instant $\mathbf{H}$ when pressure perturbations reach approximately $15 \mathrm{kPa}$, confirming the critical level of sustained pressure oscillations necessary to trigger flashback computed in Section 4 by acoustic forcing (OPEN-FO case).

This LES suggests that the flame cannot remain in the chamber for this regime and this is also what experiments showed (Section 2): it is impossible to maintain a stable flame in the chamber for these operating conditions. Flashback occurs very rapidly and the experiment must be stopped to avoid damaging the injection tube.

\section{CHOKED-ST: Control of AI-CIVB Using Fuel Staging}

The two injector stages of the test bench offer the possibility to stage both air and fuel mass flow rates. The previous analysis has shown that flashback occurs when the flame can propagate along the vortex axis. This observation was the reason why the staged case (CHOKED-ST) was tested both experimentally and numerically. For this case, fuel is entirely injected through the downstream injector (Table 1). The objective is to create a lean

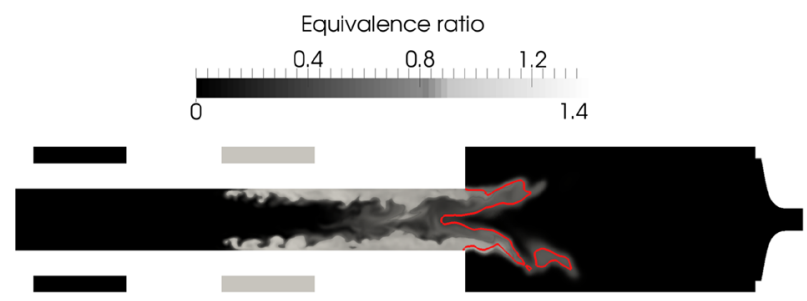

Fig. 12 Equivalence ratio $\phi$ near the dump plane in the CHOKED-ST setup, on an instantaneous field. Fuel is injected through the secondary (downstream) stage only. Solid line is a temperature isocontour at $1000 \mathrm{~K}$ 


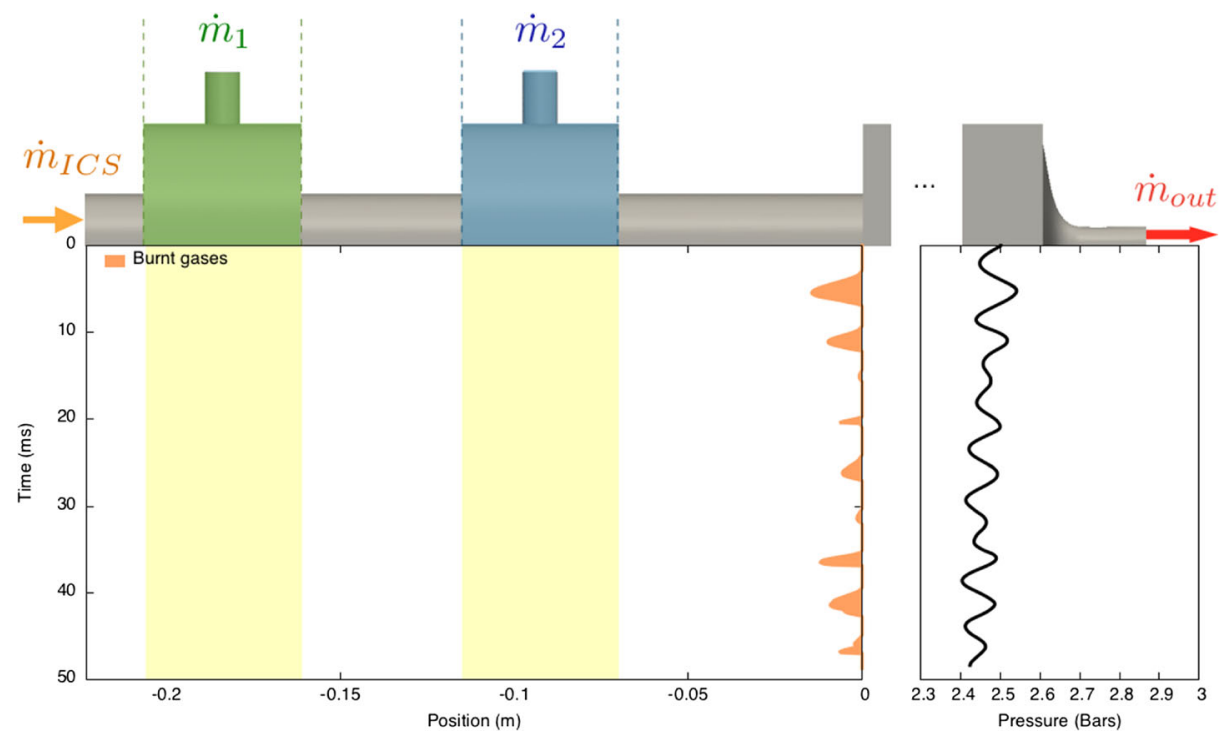

Fig. 13 Flame position and chamber pressure for the CHOKED-ST test case. $50 \mathrm{~ms}$ LES run

vortex core where flashback is inhibited. Fig. 12 provides a map of equivalence ratio $\phi$ in the CHOKED-ST case (obatined using LES). If the instability arises, the flame will be pushed into the swirl tube but flashback should not be triggered because the central zone of the injection duct is potentially lean too. A $50 \mathrm{~ms}$ LES simulation of this setup was performed. Fig. 13 shows the flame position and the domain mean pressure versus time:

- acoustic activity is still present at a frequency of $209 \mathrm{~Hz}$, similar to the LES of CHOKED-PR. However, amplitudes (less than 0.1 bar) are smaller than in the CHOKED-PR case. The instability does not grow with time anymore;

- IFB still takes place in the swirl tube in phase with pressure oscillations. However, the leading point never intrudes by more than $1.5 \mathrm{~cm}$ in the swirl tube. No flashback occurs over the course of the simulation.

\section{Conclusions}

A coupled mechanism (AI-CIVB) for flashback in swirled burners has been described, where CIVB (Combustion Induced Vortex Breakdown) is triggered by acoustic instabilities. In the swirled burner studied here, AI-CIVB causes the flame to flashback systematically when the outlet is choked because strong thermoacoustic instabilities are excited. AI-CIVB is due to the combination of strong swirl, high premixing levels and intense acoustic waves. Flashback was obtained by forcing LES acoustically in a configuration where self-excited modes were absent (OPEN-NR). In that case, an acoustic forcing amplitude of $20 \mathrm{kPa}$ was necessary to trigger full flashback, while at $10 \mathrm{kPa}$ flashback occurred only intermittently. Compressible LES was used to reproduce the acoustic behavior of the outlet nozzle. It captured the self-excited modes of the burner, and correctly predicted the experimental observations of flashback. Flashback-prone and flashback-robust configurations 
were obtained by changing fuel staging. LES recovered experimental results too, predicting which configuration is AI-CIVB resistant.

Acknowledgments This work has received funding from the ANR DISCERN program, grant ANR-11BS09-0015 of the French Agence Nationale de la Recherche. It was also supported by the European Union Seventh Framework Program (FP7/2007-2013) in the RECORD project under grant agreement $n^{0} 312444$. It was granted access to the HPC resources of CINES, CCRT and IDRIS under the allocation x20142b5031 made by GENCI (Grand Equipement National de Calcul Intensif).

\section{References}

1. Krebs, W., Flohr, P., Prade, B., Hoffmann, S.: Combust. Sci. Tech. 174, 99 (2002)

2. Lieuwen, T.: J. Prop. Power 19(5), 765 (2003)

3. Lieuwen, T., Yang, V.: in AIAA Prog. in Astronautics and Aeronautics, vol. 210, vol. 210 (2005)

4. Huang, Y., Yang, V.: Prog. Energy Combust. Sci. 35(4), 293 (2009)

5. Lewis, B., von Elbe, G.: J. Chem. Phys. 11(2), 75 (1943)

6. Plee, S., Mellor, A.: Combust. Flame 32, 193 (1978)

7. Kurdyumov, V., Fernández, E., Linan, A.: Proc. Combust. Inst. 28(2), 1883 (2000)

8. Kröner, M., Fritz, J., Sattelmayer, T.: J. Eng. Gas Turbines Power 125(3), 693 (2002)

9. Keller, J.O., Vaneveld, L., Korschelt, D., Hubbard, G.L., Ghoniem, A.F., Daily, J.W., Oppenheim, A.K.: AIAA J. 20(2), 254 (1982)

10. Thibaut, D., Candel, S.: Submitted to Combust. Flame (1997)

11. Lieuwen, T.C.: Unsteady combustor physics Cambridge University Press (2012)

12. Fritz, J., Kröner, M., Sattelmayer, T.: J. Eng. Gas Turbines Power 126(2), 276 (2004)

13. Sommerer, Y., Galley, D., Poinsot, T., Ducruix, S., Lacas, F., Veynante, D.: vol. 5 (2004)

14. Eichler, C., Sattelmayer, T.: J. Eng. Gas Turbines Power 133(1), 011503 (2011)

15. Gruber, A., Chen, J., Valiev, D., Law, C.: J. Fluid Mech. 709, 516 (2012)

16. Thibaut, D., Candel, S.: Combust. Flame 113(1), 53 (1998)

17. Syred, N., Beer, J.: Combust. Flame 23(2), 143 (1974)

18. Billant, P., Chomaz, J.M., Huerre, P.: J. Fluid Mech. 376, 183 (1998)

19. Kiesewetter, F., Hirsch, C., Fritz, J., Kroner, M., Sattelmayer, T.: In: ASME Turbo Expo 2003, collocated with the 2003 International Joint Power Generation Conference (American Society of Mechanical Engineers), pp. 293-300 (2003)

20. Kiesewetter, F., Konle, M., Sattelmayer, T.: J. Eng. Gas Turbines Power 129(4), 929 (2007)

21. Lovachev, L.: Combust. Flame 27, 125 (1976)

22. Chomiak, J.: In: Proc. Combust. Inst., vol. 16 (Elsevier), pp. 1665-1673 (1977)

23. Ashurst, W.T.: Sci, Combust. Tech 112(1), 175 (1996)

24. Umemura, A., Tomita, K.: Combust. Flame 125(1), 820 (2001)

25. Ishizuka, S., Murakami, T., Hamasaki, T., Koumura, K., Hasegawa, R.: Combust. Flame 113(4), 542 (1998)

26. Hasegawa, T., Michikami, S., Nomura, T., Gotoh, D., Sato, T.: Combust. Flame 129(3), 294 (2002)

27. Hasegawa, T., Nakamichi, R., Nishiki, S.: Combust. Theor. Model. 6(3), 413 (2002)

28. Domingo, P., Vervisch, L.: Proc. Combust. Inst. 31(1), 1657 (2007)

29. Tran, N., Ducruix, S., Schuller, T.: In: 13th AIAA/CEAS Aeroacoustics Conference, Paper No AIAA2007-3716 (2007)

30. Tran, N., Ducruix, S., Schuller, T.: Proc. Combust. Inst. 32(2), 2917 (2009)

31. Scarpato, A., Tran, N., Ducruix, S., Schuller, T.: J. Sound Vib. 331(2), 276 (2012)

32. Reichel, T.G., Terhaar, S., Paschereit, O.: In: Proceeding of ASME Turbo Expo 2014: Turbine Technical Conference and Exposition, vol. 4B (American Society of Mechanical Engineers) (2014)

33. Sattelmayer, T., Mayer, C., Sangl, J.: In: Proceeding of ASME Turbo Expo 2014: Turbine Technical Conference and Exposition, vol. 4A (American Society of Mechanical Engineers (2014)

34. Brouckaert, J.F., Mersinligil, M., Pau, M.: Proceedings of the ASME Turbo Expo 2008 Power for Land, Sea and Air (2008)

35. Mersinligil, M., Brouckaert, J.F., Desset, J.: Proceedings of the ASME Turbo Expo 2010 Power for Land, Sea and Air (2010)

36. Mersinligil, M., Desset, J., Brouckaert, J.F.: Proceedings of the Institution of Mechanical Engineers, Part A. J. Power Energy (2011) 
37. Guin, C.: RTO Meeting proceedings (1999)

38. Burmberger, S., Sattelmayer, T.: J. Eng. Gas Turb. and Power (2011)

39. Beér, J.M., Chigier, N.A.: Combustion aerodynamics. (robert e krieger publishing company) (1972)

40. Noble, D.R., Zhang, Q., Shareef, A., Tootle, J., Meyers, A., Lieuwen, T.: (2006)

41. Beerer, D., McDonnel, V., Therkelsen, P., Cheng, R.K.: Eng, J. Gas turb and power 136, 031502-1 (2014)

42. Schönfeld, T., Rudgyard, M.: AIAA J. 37(11), 1378 (1999)

43. Selle, L., Lartigue, G., Poinsot, T., Koch, R., Schildmacher, K.U., Krebs, W., Prade, B., Kaufmann, P., Veynante, D.: Combust. Flame 137(4), 489 (2004)

44. Colin, O., Rudgyard, M.: J. Comput. Phys. 162(2), 338 (2000)

45. Poinsot, T., Lele, S.: J. Comput. Phys. 101(1), 104 (1992). doi:10.1016/0021-9991(92)90046-2

46. Granet, V., Vermorel, O., Leonard, T., Gicquel, L., Poinsot, T.: AIAA J. 48(10), 2348 (2010)

47. Nicoud, F., Baya Toda, H., Cabrit, O., Bose, S., Lee, J.: Phys. Fluids 23(8), 085106 (2011). doi:10.1063/1.3623274. http://link.aip.org/link/?PHF/23/085106/1

48. Charlette, F., Meneveau, C., Veynante, D.: Combust. Flame 131, 159 (2002)

49. Lapeyre, C.J.: Numerical study of ame stability, stabilization and noise in a swirl-stabilized combustor under choked conditions. Ph.D. thesis, INP Toulouse (2015)

50. Tsien, H.S.: SIAM J. Appl. Math. 6, 188 (1951)

51. Kitoh, O.: J. Fluid Mech. 225, 445 (1991)

52. Ishizuka, S.: Prog. Energy Combust. Sci. 28(6), 477 (2002) 